\title{
¿Qué relación guarda la motivación con los problemas de disciplina durante la adolescencia en los programas de educación bilingüe en EEUU?
}

\author{
María Martínez Lirola \\ Universidad de Alicante
}

Recibido: 2 marzo 2004 / Versión aceptada: 7 junio 2004

\begin{abstract}
RESUMEN: El objetivo principal de este artículo es poner de manifiesto que la baja motivación y el factor edad genera en los adolescentes actitudes agresivas y comportamientos violentos en los institutos. Nuestro estudio se basa en la observación de estudiantes mexicanos de enseñanza secundaria bilingüe, en concreto en la asignatura Bilingual Language Arts en los niveles 10, 11 y 12.

Los inmigrantes en Estados Unidos tienen necesidades especiales en la educación y los programas de educación bilingüe tratan de dar respuesta a esas necesidades. El objetivo principal de estos programas es facilitar el aprendizaje del inglés, así como mantener la lengua y la cultura materna, en este caso el español.

Enseñar a estudiantes adolescentes entre 15 y 19 años llevaba consigo que no estuvieran motivados para aprender. Esta falta de motivación creaba problemas de agresividad en las aulas. La edad es un factor determinante en la enseñanza de adolescentes y en el proceso de aprendizaje debido a que a esta edad los alumnos no son muy receptivos a aprender otras lenguas.
\end{abstract}

Palabras clave: educación Bilingüe, motivación, disciplina, factor edad, aprendizaje.

ABSTRACT: The main objective of this paper is to point out that low motivation and age create aggressive attitudes and violent behaviour in adolescents at high school. This study is based on the observation of Mexican students of a bilingual education program in the United States. They were students of Bilingual Language Arts at grades 10,11 and 12.

Immigrants in the United States have special necessities in education and bilingual education programs try to be an answer to those needs. The main objective of these programs is to make easier the apprenticeship of English, together with the maintenance of the maternal language and culture, in this case Spanish.

The fact of teaching adolescents between 15 and 19 years old implied that their motivation was hardly present in the learning process. This lack of motivation created aggressive behaviours in the students. Age is a determined factor in adolescents' teaching and learning process since at this age students are not very receptive to learn other languages and cultures.

Key words: bilingual education, motivation, discipline, age factor, learning. 


\section{INTRODUCCIÓN}

Es un hecho que el español se ha convertido en segunda lengua obligatoria en los institutos norteamericanos y su presencia se extiende a lo largo y ancho del país. Por este motivo, el Ministerio de Educación y Ciencia tiene un convenio con EEUU para proporcionarle profesores nativos de español con el fin de que los alumnos aprendan y perfeccionen esta lengua.

Este artículo viene motivado por el año pasado como profesora de enseñanza secundaria gracias a este convenio en un instituto de Estados Unidos, en concreto en Deming, Nuevo México. La asignatura impartida se denomina Bilingual Language Arts y consiste en introducir al alumno en la literatura en lengua española y en lengua inglesa, así como en cuestiones gramaticales en ambas lenguas, de modo que el alumno desarrolle las diferentes destrezas comunicativas.

Dicha asignatura se enmarca dentro del Programa de Educación Bilingüe del centro de enseñanza. Estos programas tienen como base el respeto a la diversidad cultural y tienen en cuenta la igualdad de derechos y deberes entre todos los miembros de las diferentes culturas. El objetivo prioritario es facilitar el aprendizaje de la lengua inglesa junto con el mantenimiento de la lengua y cultura maternas, en este caso la española. De este modo vemos claramente una de las funciones principales de la educación: desarrollar el diálogo entre culturas que fomente la comunicación y la solidaridad. Se pretende evitar que el grupo mayoritario (la sociedad estadounidense) absorba al minoritario (los inmigrantes mexicanos) para que este último no pierda su identidad y mucho menos su lengua. Uno de los objetivos fundamentales es que los miembros del grupo mayoritario acepten como iguales a los de grupos minoritarios.

Es evidente que las escuelas e institutos tienen dos funciones principales: por un lado han de socializar, es decir, han de transmitir pautas de comportamiento y por otro lado han de enseñar, es decir, transmitir conocimientos y habilidades.

La mayoría de los alumnos que formaban parte del programa bilingüe eran hijos de inmigrantes mexicanos que estudiaban en EEUU para tener una mejor educación y poder estudiar en dos lenguas a la vez: el español, su lengua materna, y el inglés, la lengua del país en que residen.

A nuestro juicio, este estudio es pertinente en el contexto español actual porque se está fomentando la introducción de programas de educación bilingüe en enseñanza primaria y secundaria, especialmente en las comunidades autónomas en las que el español convive con otras lenguas.

Nuestro objetivo principal con este artículo es poner de manifiesto que la falta de motivación en los alumnos adolescentes que formaban parte de un programa de educación bilingüe creaba problemas de agresividad en las aulas. La edad es un factor determinante en la enseñanza de adolescentes y en el proceso de aprendizaje debido a que a esta edad los alumnos no son muy receptivos a aprender otras lenguas.

El instrumento empleado para obtener los datos a los que nos referiremos en los apartados posteriores ha sido la observación. 
María Martínez Lirola ¿ ¿Qué relación guarda la motivación con los problemas de disciplina ...

\section{Características de los programas de educación bilingüe en EeUU, CON ESPECIAL REFERENCIA A lOS PROGRAMAS biLINGÜES EN ENSEÑANZA SECUNDARIa}

La educación bilingüe en Estados Unidos surgió en los años sesenta y llevaba consigo la demanda y los intereses de la población hispana. Los programas de educación bilingüe surgen para dar respuesta a las necesidades de los hijos de los trabajadores inmigrantes.

El término bilingüismo ha sido siempre polémico y de él se han ofrecido numerosas definiciones. Nosotros usaremos el término bilingüe para indicar el uso de dos idiomas con diferentes propósitos.

Introducir a estos jóvenes en el aprendizaje de otra lengua lleva consigo que los alumnos aprendan no sólo a usar reglas de gramática, fonética y vocabulario sino que también implica un tipo de comportamiento no verbal, como por ejemplo los gestos, de ahí que podamos referirnos a la hipótesis Sapir-Whorf (1956), según la cual aprender una lengua nueva lleva consigo un cambio en el modo de pensar, es decir, hay una serie de cambios no verbales.

Dada la importancia de los programas de educación bilingüe, es fundamental que cumplan los siguientes requisitos:

1. Responder a las necesidades educativas de estudiantes cuya lengua materna y cuya cultura es diferente a la de la mayoría. Estas respuestas irán presididas por un programa de desarrollo de la lengua inglesa.

2. Usar dos lenguas en todas las materias del currículum, en todos los niveles en los que el alumno forme parte del programa.

3. Los profesores han de ser perfectos conocedores de las dos lenguas que trata de desarrollar el programa.

4. Enfatizar la historia y las culturas asociadas con la lengua materna de los alumnos e incluir éstas en el currículum.

5. Todo esto ha de desarrollarse bajo la supervisión de un plan estatal.

Los programas de educación bilingüe pueden ser de tres tipos principales siguiendo a Hamers y Blanc (1983:301): por un lado la enseñanza puede darse en las dos lenguas; por otro lado la enseñanza puede darse en la lengua materna del alumno hasta que esté en condiciones de seguir sus estudios en la segunda lengua, de este modo los conocimientos que adquiere en su lengua materna le ayudarán a que el inglés que escuchan y leen sea más comprensible; finalmente la mayor parte de la enseñanza puede darse en la segunda lengua de los alumnos, empleándose la lengua materna sólo ocasionalmente.

Un componente esencial en estos programas consiste en desarrollar en el alumno sus destrezas de lectura y escritura en la lengua materna. Esta lengua se utiliza para clarificar situaciones de modo que el alumno pueda comprender los mensajes de un modo claro y preciso. Otro componente fundamental es el aprendizaje de un modo estructurado del inglés como segunda lengua. Tanto los alumnos monolingües como los hablantes parciales de inglés usarán métodos y materiales designados para ello. Estos métodos harán que su comprensión y su modo de hablar, leer y escribir sean cada vez mejores. El programa ha de centrarse en los aspectos sociales, culturales, económicos e históricos de la cultura de los alumnos. 
El Ministerio de Educación especifica que los programas de educación bilingüe se establecen para dar respuesta a las necesidades educativas y lingüísticas de los estudiantes, siendo prioritarios los estudiantes cuya segunda lengua es el inglés. La instrucción ha de llevarse a cabo cuarenta y cinco minutos en la lengua materna y cuarenta y cinco minutos en la lengua extranjera, en este caso el inglés. Esto implica que la evaluación del alumno se llevará a cabo tanto en inglés como en la lengua materna.

Es evidente que este tipo de programas educativos requieren lo que se denomina un profesor bilingüe, es decir, hablantes de lengua inglesa y de la lengua materna de la comunidad minoritaria (en nuestro caso español). Estos profesores deben desarrollar las destrezas de los alumnos en la lengua materna y ser conscientes de las diferencias y semejanzas que existen entre ésta y la lengua extranjera, adentrarse en su contexto social, cultural y educativo, así como exponer a los alumnos a la lengua inglesa. Es esencial que el profesor tenga presente que el alumno que aprende inglés no es sólo alguien que no habla inglés bien sino alguien que ha crecido en el contexto de una cultura diferente.

El profesor es el modelo para los estudiantes en la clase y tiene un doble papel: por un lado ha de ayudar al alumno a mejorar su nivel de inglés y hacer que se sienta integrado en el centro; por otro lado ha de ayudar a los alumnos a aceptar y comprender la riqueza inherente en diferentes lenguas, en diferentes culturas.

La situación a veces es complicada porque muchos de los alumnos son inmigrantes recién llegados que no poseen destrezas en la lengua inglesa, mientras que otros sí han estudiado inglés antes de llegar a los Estados Unidos. En algunos casos el problema es aún más grave porque el profesor puede encontrarse con alumnos que nunca han estado escolarizados o durante muy poco tiempo, hecho que hace que sus destrezas en la lengua materna estén muy limitadas por lo que no le pueden ayudar a mejorar la segunda lengua que en este caso es el inglés. El profesor debe dedicar un tiempo a investigar las culturas de las que proceden sus alumnos de modo que pueda comprender mejor algunos modos de actuación de éstos, por ejemplo, en algunas culturas los alumnos sólo hablan cuando se les pregunta; nuestros alumnos presentaban buenas destrezas orales mientras que carecían de habilidades para comprender nuevos conceptos por medio de la lengua escrita.

Encontramos alumnos hispanos con un buen nivel de inglés oral pero con muchas dificultades a la hora de leer y/o escribir en dicha lengua. Algunos alumnos, muy acostumbrados a emplear la lengua materna, la mezclan con la lengua inglesa y carecen de destrezas comunicativas en las dos lenguas. El papel del profesor ante estas situaciones ha de consistir en ayudar a los alumnos a mejorar su nivel de la lengua materna y en la lengua inglesa, de modo que se le pueda denominar bilingüe

Estos programas de educación bilingüe se justifican porque las necesidades del grupo de población mayoritario son diferentes a las de la minoría. En caso de no existir, los grupos de población minoritaria estarían en desventaja por carecer de los conocimientos de inglés necesarios para seguir un programa de educación regular.

Para que los programas de educación bilingüe sean buenos han de tener los siguientes componentes (Krashen, 1996:4):

1. Deben ofrecer al alumno información en inglés

2. Deben ofrecer parte de la materia en la lengua materna del alumno, sin ningún tipo de traducción. Esta ayuda indirecta ayuda a que los alumnos lean y entiendan mejor la lengua inglesa. 
María Martínez Lirola ¿Qué relación guarda la motivación con los problemas de disciplina ...

3. Enseñará las destrezas de lectura y escritura en la lengua materna, de modo que el alumno pueda aplicarlas a la lengua inglesa.

4. Un cuarto componente es el continuo desarrollo de la lengua materna (L1).

El propósito que ha de cumplir este tipo de programa de educación ha de ser doble son los siguientes: por un lado han de estar en consonancia con las experiencias de la comunidad; por otro lado han de fomentar habilidades en los alumnos que puedan ser emepleadas en el mundo actual (Di Pietro: 1978:107)

Hamers y Blanc (1983:302) consideran como fin principal de este tipo de educación lo siguiente: compensar entre la lengua dominante (la de la mayoría) y la lengua dominada (la de la minoría). El programa le permitirá aprender en la lengua que le es familiar para progresar rápidamente es la lengua de la mayoría; en segundo lugar, los programas permiten el enriquecimiento lingüístico de los estudiantes del grupo de población mayoritaria; finalmente pretende mantener la lengua y la cultura de la minoría, ofreciendo la posibilidad de participar en las actividades del grupo de población dominante.

Los patrones educativos y sociolingüísticos en los que se enmarca el programa tienen importantes consecuencias en el éxito o fracaso del mismo, es decir, el programa se ve afectado por factores psicológicos, sociológicos, económicos, políticos, religiosos, culturales, geográficos y lingüísticos tanto del grupo minoritario como del grupo cuya lengua materna es la inglesa.

\section{Principales dificultades en la enseñanza: experiencias}

Aprender una lengua no es simplemente cuestión de inteligencia. La persona ha de desarrollar una serie de habilidades fonéticas, gramaticales, memorísticas, etc. que le permitan reconocer y repetir sonidos, inferir reglas y generalizaciones gramaticales, hacer asociaciones entre las palabras, etc.

La edad y la motivación son dos factores que están claramente presentes en los alumnos. Es evidente que la edad es un factor fijo pues no depende del control externo mientras que la motivación es un factor que varía con el tiempo, tal y como expone Ellis (1994: 35-36): "Age is an example of a fixed factor, in the sense that it is beyond external control. [...] Motivation is an example of a factor that is clearly variable. The strength of an individual learner's motivation can change over time and is influenced by external factors".

\subsection{El factor edad en las aulas}

La edad de los alumnos es un factor determinante en la enseñanza de las lenguas extranjeras. En nuestro caso, nos encontramos con alumnos de los últimos cursos de enseñanza secundaria (grados 10, 11 y 12) con edades comprendidas entre los quince y los diecinueve años.

La adolescencia ha de considerarse un período crítico para el aprendizaje y la enseñanza de lenguas extranjeras ya que el conocimiento del mundo de los adolescentes y los cambios físicos y psicológicos a los que se enfrentan a esta edad, centrados sobre todo en un alto interés en crear y mantener una identidad personal hacen que el input en la lengua extranjera no pueda integrarse de manera acertada ya que en los adolescentes predominan sentimientos 
de vulnerabilidad, baja autoestima, etc. que lo único que hacen es interponerse con el aprendizaje de la lengua extranjera.

Los adolescentes tienen como principal interés su propia identidad y piensan que el resto de la gente comparte este interés. La adolescencia se caracteriza por ser un período de inestabilidad emocional, hipersensibilidad y egocentrismo que lleva a los alumnos a pensar que todo el mundo está pendiente de ellos de modo que se manifiesta una gran vulnerabilidad. En general están poco abiertos a nuevas personas y experiencias que representan un riesgo y un miedo al ridículo.

Lo expuesto en párrafos anteriores no lleva consigo que durante la adolescencia cambie el proceso de adquisición de lenguas aunque, según exponen algunos autores como Krashen y Terrell (1988:47), parece ser que hay un bloqueo en la adquisición, como podemos observar en la siguiente afirmación: "[...] there is no fundamental change in the language acquisition process at puberty. The ability to acquire does not disappear at puberty nor is it seriously damaged; rather, the necessary input is often blocked and therefore is less available for acquisition."

\subsubsection{El factor edad como responsable de la falta de motivación}

La motivación o deseo de aprender es un factor fundamental en el aprendizaje; podemos considerarla como uno de los aspectos más importantes en el proceso educativo. Se trata de un factor psicológico que pone de manifiesto el éxito de los estudiantes a la hora de aprender una lengua extranjera. Nos atrevemos a afirmar que los alumnos con un alto grado de motivación aprenden antes y obtienen mejores resultados en el aprendizaje de una lengua extranjera.

La motivación se caracteriza por una serie de rasgos como son la agencialidad, es decir, con la intencionalidad para hacer algo, la activación o predisposición para hacer o aprender algo, la participación de los afectos ya que normalmente queremos conseguir cosas que nos gustan, y finalmente suele estar dirigida a una meta bien por utilidad personal o por necesidades sociales. Es evidente que podemos aprender cosas sin querer, es decir, hay aprendizajes en los que no hay motivación y por el contrario, a veces hay motivación y no hay aprendizaje, sólo hay divertimento. Lo ideal es que aprendizaje y motivación vayan unidos para que se produzcan aprendizajes significativos.

Aunque en general observamos falta de motivación en los alumnos observados, es fundamental destacar que observamos distintos tipos de motivación en los alumnos dependiendo de las actividades propuestas en clase por lo que en los párrafos siguientes vamos a presentar los distintos tipos de motivación.

Basándonos en las investigaciones de Gardner y Lambert $(1959,1972)$ podemos hablar de motivación integradora que permite al individuo estudiar la lengua como algo apetecible al mismo tiempo que se identifica e integra con la cultura de esa lengua. Ellis (1994:513) pone de manifiesto que los alumnos en los que encontramos este tipo de motivación son más activos en clase y es menos probable que abandonen.

La mayoría de los alumnos observados carecían de este tipo de motivación porque ellos no habían elegido estudiar en un programa de educación bilingüe sino que había sido una decisión de sus familias.

Por otra parte podemos hablar de motivación instrumental que se refiere a las consideraciones prácticas y se relaciona con las ventajas que aprender esa lengua proporciona 
María Martínez Lirola ¿ ¿Qué relación guarda la motivación con los problemas de disciplina ...

al individuo, por ejemplo, un individuo puede aprender una lengua para pasar un examen, conseguir un buen trabajo, etc.

Nuestros alumnos sí tenían motivación instrumental pues la mayoría de ellos pretendían conseguir trabajo en EEUU, de modo que pudiera mejorar la calidad de vida de sus familias. Cuando los alumnos aprenden por incentivos como el dinero, normalmente aprenden más rápidamente, tal y como afirma Ellis (1994: 514):

[...] learners with an instrumental reason for learning an L2 can be successful. In some 'second' as opposed to 'foreign' settings an instrumental orientation may be the most important one. Providing learners with incentives (such as money) may also aid learning by increasing the time learners spend studying, but the effects may cease as soon as the reward stops.

La motivación extrínseca se basa en factores externos, es decir se relaciona con los materiales y el método de enseñanza mientras que la motivación intrínseca se basa en factores internos al individuo.

Es evidente que como profesores somos responsables de la motivación extrínseca, pues es evidente que este tipo de motivación puede aumentar en los alumnos si empleamos un método comunicativo y unos materiales que ayuden al alumno a relacionar lo aprendido en clase con su vida diaria.

Pavón Vázquez (2001:171) señala que en el aprendizaje de una lengua extranjera existe por un lado un condicionante fisiológico, por lo que el dominio del aprendiz se desarrolla a partir de la influencia de los hábitos de producción para la lengua materna y por la influencia que ejerce la segunda lengua y el proceso mismo de adquisición/aprendizaje. En la misma página, Pavón Vázquez pone de manifiesto que una segunda explicación de tipo psicológico propugna que la formación de hábitos en la lengua materna y los procesos que intervienen en su adquisición le crean al aprendiz una capacidad de origen psicológico para alterar los criterios de utilización lingüística, que son diferentes en la segunda lengua. La tercera explicación que el autor propone para la adquisición/aprendizaje de una lengua extranjera enfatiza la importancia de la empatía del aprendiz con la segunda lengua, con sus hablantes y con su cultura.

La afirmación anterior nos lleva a poner de manifiesto que los estudiantes mexicanos a los que impartimos clase tenían poca competencia tanto en inglés como en su lengua materna y su grado de empatía con los hablantes y cultura norteamericana era bastante bajo. Es evidente que una actitud negativa hacia la lengua extranjera o hacia sus hablantes afecta seriamente al aprendizaje ya que la actitud que el aprendiz muestra hacia la lengua extranjera que está aprendiendo y hacia los hablantes nativos de esa lengua afecta claramente a que alcance un éxito determinado.

No hemos de olvidar que el éxito en el aprendizaje de una lengua extranjera también se relaciona con la edad en que empiezan los individuos a tener contactos con la segunda lengua, pues los que empiezan a una edad temprana suelen adquirir una pronunciación bastante cuidada. Según la hipótesis del período crítico, a una cierta edad es más difícil aprender una lengua, tal y como exponen Gass y Selinker (2001:335) en que:

It is commonly believed that children are better language learners than adults in the sense that young children typically can gain mastery of a second language, 
whereas adults cannot. This is reflected in what is known as the Critical Period Hypothesis (or, Sensitive Period Hypothesis). According to this hypothesis, there is an age-related point (generally puberty) beyond which it becomes difficult or impossible to learn a second language to the same degree as NSS ${ }^{l}$ of that language.

Muchos autores ponen de manifiesto que después de la pubertad es más difícil alcanzar un nivel avanzado en lengua extranjera y tener un buen acento en dicha lengua debido a que la pubertad hace que el individuo se centre en la consolidación de su personalidad y no conceda importancia a las normas que el aprendizaje de una segunda lengua requiere (Larsen y Smalley,1972:160).

La motivación es uno de los factores principales que influyen en el aprendizaje de una lengua; de manera similar es habitual encontrar problemas de disciplina cuando el alumno carece de motivación y las estrategias que el profesor emplea en sus clases no se corresponden con los intereses de los alumnos.

Los alumnos que realmente tienen interés por aprender, aprenden independientemente de las circunstancias que les rodeen. En el proceso de aprendizaje juega un papel fundamental la motivación, una especie de motor interno, una fuerza que impulsa a las personas a llevar a cabo una acción, a conseguir un objetivo.

\subsubsection{El factor edad como responsable de los graves problemas de disciplina}

El hecho de que la mayoría de los alumnos a los impartimos clase en EEUU fueran adolescentes con una baja motivación para aprender llevaba consigo que los alumnos desarrollaran en muchas ocasiones actitudes violentas en el aula. Es decir, muchos alumnos no sólo se negaban a aprender lengua inglesa y española sino que se rebelaban contra el aprendizaje por medio de conductas agresivas. Es evidente que el término violencia se relaciona de un modo estrecho con el comportamiento y la mala conducta. Las definiciones de Charles (1991:vi) nos parecen clarificadoras:

Behavior is defined as all the physical and mental acts that human performs. Thus behavior is whatever one does, whether good or bad, right or wrong, helpful or useless, productive or wasteful. In contrast, misbehavior is a label applied to any behaviour that is considered to be inappropriate to the setting or situation in which it occurs. Most classroom misbehavior is considered to be done intentionally by students, when they know they should not do it.

En la misma página el autor establece que hay cinco tipos de mala conducta:

1. Agresión: ataques físicos o verbales llevados a cabo por el alumno contra el profesor u otros estudiantes.

2. Inmoralidad: engloba actos como copiar en exámenes, mentir o robar.

3. Desafío a la autoridad: ocurre cuando el alumno se niega a hacer lo que el profesor le pide.

${ }^{1}$ NSS aparece en lugar de hablantes nativos (native speakers). 
María Martínez Lirola ¿Qué relación guarda la motivación con los problemas de disciplina ...

4. Interrupciones en la clase: hablar en alto, caminar en la clase, lanzar objetos, etc.

5. Eludir las responsabilidades al no hacer sus tareas, mantenerse pensando en otros temas, etc.

Es evidente que la mala conducta nos lleva a hablar de disciplina, es decir, de las medidas que es necesario tomar para que los alumnos se comporten en clase de la manera correcta, de modo que respeten al resto de los compañeros y al profesor/a. Es obvio que la disciplina tiene como propósito controlar y suprimir la mala conducta. Charles (1991:vii) también señala que la disciplina en el aula lo que pretende es reducir la necesidad de que el profesor intervenga en exceso para que los alumnos controlen su propia conducta.

Nos parece sorprendente que en los Estados Unidos, una actividad tan noble como educar que consiste en que la persona se desarrolle y se enriquezca, sea capaz de sacarle el máximo partido a su espacio y su tiempo, etc. esté cada vez más amenazada por la violencia.

Martínez Sánchez (2001:63) cita algunos titulares de diferentes periódicos de todo el mundo aparecidos en los últimos años en los que la violencia parece ser algo habitual: "Castigos corporales a los estudiantes"; "De maltratado a maltratante"; "La violencia y la escuela"; "Estudias o destrozas"; "Un decreto ministerial recogerá el derecho de los alumnos a no recibir castigos físicos o morales"; "Falta respeto y disciplina en las aulas"; "Educación y castigos"; "Aumentan los brotes de violencia en las aulas"; "La calle irrumpe en las aulas"; "Los brotes de agresividad entre adolescentes ponen en alerta a los centros escolares", etc.

Si nos planteamos cuál es la causa de que la violencia sea uno de los problemas más importantes en las escuelas e institutos estadounidenses hemos de referirnos a los valores que priman en todos los ámbitos de la sociedad: un valor importante es tener éxito, triunfar; para ello es necesario competir, aparentar o luchar por ser más que los demás, etc. Este valor nos lleva al siguiente: el individualismo, como valor opuesto a la solidaridad. Parece que el éxito o el fracaso están ligados a cada uno sin prestar mucha atención a los factores sociales, culturales o institucionales que evidentemente llevan consigo una responsabilidad.

La afirmación anterior nos lleva a señalar que prima el tener frente al ser. Lo importante es poseer por lo que en la actualidad un buen grupo de la población se deja llevar por un consumo desmesurado. Los que no pueden poseer todo lo que desean a veces caen en la delincuencia o en la violencia al no poder comprar todo lo que les gustaría como hacen las clases privilegiadas. Esto hace que el valor de una persona esté en su nivel de ingresos: una persona vale según el lugar en el que vive, el coche que tiene, la ropa que lleva puesta, etc. Lo que importa es la apariencia, la imagen, la utilidad. Parece que es más importante ser un cliente que ser un ciudadano, porque lo que importa es la capacidad de compra que una persona tiene y que se puede resumir muy bien en la frase "tanto tienes, tanto vales".

Lo anteriormente expuesto nos lleva a declarar que es más importante aparentar que ser; el ser está sustituido por el tener y el tener por el aparentar. Nos encontramos en una época de relativismo irreflexivo, en el que parece que todo vale si produce beneficios. Todo esto parece apuntar a que son pocas las ideologías totalitarias; no hay una ética sino microéticas ya que al primar el individualismo y ser el centro uno mismo, cada uno tiene su propia ética que condiciona su modo de actuación.

Estamos de acuerdo con Martínez Sánchez (2001:75) en que la violencia está tan presente en las escuelas y en los institutos norteamericanos y en todas los ámbitos de la sociedad en general porque en la sociedad hay desigualdades e injusticias de todo tipo, hay un desencanto y pesimismo generalizado que se agudiza especialmente entre los jóvenes. 


\subsubsection{Medidas disciplinarias}

Una visión social de la lengua nos hace conscientes del contexto sociolingüístico de los alumnos que son objeto de estudio: en nuestro caso nos encontramos con alumnos de los últimos cursos de enseñanza secundaria (grados 10, 11 y 12) con edades comprendidas entre los quince y los diecinueve años; todos ellos son mexicanos, hijos de trabajadores que han emigrado a EEUU para trabajar y ofrecer a sus hijos un futuro mejor en los Estados Unidos.

Desde esta perspectiva social, siempre hemos de considerar que cuando surgen problemas de disciplina hemos de tener siempre presentes factores como: el contexto que envuelve a los alumnos, sus diferentes personalidades así como la personalidad del profesor, su filosofía personal de la educación y su conocimiento de la cultura de los alumnos.

La falta de disciplina también pone de manifiesto en muchas ocasiones un problema de autoestima ya que algunos alumnos en vez de irradiar sentimientos y actitudes positivas hacia sí mismos, desarrollan frustraciones y poca valoración de sí mismos que en muchas ocasiones se manifiesta por medio de actitudes violentas.

La disciplina se relaciona también claramente con un problema de autoridad, es decir, el profesor ha de ser capaz de que los alumnos sigan las instrucciones que les propone para que el proceso educativo pueda tener lugar.

En las escuelas e institutos norteamericanos es normal comenzar el año estableciendo unas normas de clase que servirán de referencia a los alumnos y profesores. El profesor puede sugerir algunas pero lo normal es que las negocie con los alumnos de modo que ellos se sientan parte importante de la clase y asuman de este modo su responsabilidad. En nuestro caso, les invitábamos a que escribieran en una cartulina las normas que ellos veían como fundamentales para la convivencia en el aula. Después, entre todos, elegíamos las fundamentales procurando que no fueran más de cinco o seis normas. Las normas más habituales solían ser: respetar a los demás, hacer las tareas, traer y cuidar el material, usar el lenguaje correcto y no comer ni beber en clase.

En todo momento intentamos que las normas de clase fueran vistas por los alumnos como algo importante en el aula. De igual modo tratamos que fueran lógicas, con sentido común y que su enunciado estuviera expresado de la manera más positiva posible.

Sin duda, es fundamental que los docentes dediquemos nuestra energía no tanto a aplicar medidas disciplinarias sino a prevenir los problemas de disciplina en el aula. El profesor debe hacer un gran esfuerzo por observar a los alumnos constantemente, de modo que si surgen algunos problemas de disciplina, puedan solucionarse rápidamente.

Cuando los problemas no eran serios, intentábamos siempre solucionarlos por medio del contacto ocular o de las expresiones faciales, de modo que el alumno fuera consciente de que la profesora había detectado un problema en su comportamiento y quería que se solucionara de inmediato. En otras ocasiones nos acercábamos al alumno que estaba alterando el orden normal de la clase y sin regañarle, tratábamos que la mera presencia del profesor le sirviera para reaccionar.

Siguiendo a Prodromou (1995), ponemos de manifiesto algunas medidas para intentar controlar los problemas de disciplina en las aulas. Todas ellas las pusimos en práctica durante nuestra experiencia docente en EEUU: 
María Martínez Lirola ¿Qué relación guarda la motivación con los problemas de disciplina ...

1. La primera medida que un docente ha de adoptar cuando surge algún problema de disciplina, ha de ser ponerlo de manifiesto en la clase por medio de su tono de voz o de la mirada. Esta técnica tan sencilla es a veces muy eficaz.

2. En las escuelas e institutos americanos es muy común lo que se denomina detención (detention): es decir, el alumno se queda después del horario oficial de clase haciendo alguna tarea extra. Esto tiene un gran inconveniente para el docente, ya que también ha de dedicar parte de su tiempo libre.

3. Otro remedio común para los problemas de disciplina es anunciar un examen sorpresa. Normalmente esto hace que los alumnos reflexionen rápidamente.

4. Es también habitual cambiar los sitios de los alumnos, de modo que los más problemáticos no se sienten juntos y puedan aprender buenos hábitos de otros compañeros de clase si comparten asientos con ellos.

5. Cuando hay un problema de disciplina en el aula, a veces se puede controlar cambiando la actividad. Hay actividades, sobre todo las comunicativas, que se prestan más a que los alumnos no tengan en cuenta la compostura. Cuando esto ocurre, inconscientemente, los alumnos pueden asociar la nueva actividad a una nueva manera de comportarse.

6. También es muy habitual en EEUU que el profesor llame al alumno y tenga con él una pequeña conversación en la que le invita a cambiar su comportamiento y le advierte de que si continúa así, llamará a sus padres. Ésta es otra medida que se lleva a la práctica si el problema persiste, aunque la experiencia nos dice que en la mayoría de las ocasiones los padres manifiestan que estos alumnos conflictivos también tienen problemas en casa y ellos no saben cómo actuar para solucionar el problema.

7. Otra posibilidad con la que cuenta el docente consiste en acudir a la institución educativa, dirigiéndose a personas como el jefe de estudios o el director del centro. Antes de tomar esta medida es siempre conveniente comentar los problemas de disciplina con otros profesores, de modo que ellos puedan compartir con nosotros las técnicas que ellos utilizan para controlar su clase.

Independientemente de la medida para controlar la disciplina por la que optemos, siempre hemos de actuar inmediatamente, de modo que el docente pueda poner de manifiesto su autoridad rápidamente.

Tras haber expuesto estas medidas para controlar la disciplina, nos parece importante destacar las que consideramos más eficaces basándonos en la edad del alumnado: suele ser muy efectivo regañar a los alumnos delante de sus compañeros pues para los adolescentes es fundamental sentirse parte del grupo de iguales, de modo que poner su comportamiento violento delante de los demás les hace sentirse diferentes, sienten que no pertenecen al grupo y suelen comportarse adecuadamente. La medida del examen sorpresa también era bastante efectiva con nuestros alumnos debido a que no suelen sentirse preparados para hacerlo y acceden a portarse bien si el examen no tiene lugar en ese momento. Finalmente, de las siete medidas propuestas, fue muy eficaz cambiar la actividad que se estuviera realizando por otra distinta. Es bueno pasar de una actividad comunicativa a otra en la que los alumnos tengan que trabajar de manera individual, como por ejemplo cambiar de una actividad oral a un ejercicio escrito. 


\section{UNA ALternativa: LA EDUCACIÓN PARA LA PAZ Y LA NO-VIOLENCIA}

La violencia es lo opuesto a una cultura de la paz y la no-violencia que se caracteriza por el rechazo a la violencia en todas sus formas, y la defensa de la solidaridad, la justicia y la tolerancia, valores por medio de los cuales trata de prevenir los conflictos en sus raíces.

Optar por una cultura de la paz y la no-violencia lleva consigo la afirmación de que la violencia no es innata en el ser humano y por lo tanto es evitable. Ésta es una visión de la paz negativa, que se caracteriza por la ausencia de conflictos. La paz positiva se caracteriza no sólo por oponerse a la violencia sino también por integrar la comprensión, el respeto, la tolerancia, etc. con el fin de hacer realidad una convivencia presidida por esos valores.

La paz ha de ser un proceso dinámico que renuncia a la violencia como forma de resolver los conflictos, tomando conciencia de las situaciones y planteando formas alternativas para solucionarlos de manera justa. La paz verdadera va unida a la justicia, a la defensa de los derechos humanos y al respeto a la diversidad. Ni que decir tiene que las aulas son el escenario perfecto para poder integrar todos esos valores.

Educar para la cultura de la paz es un desafío. El primer paso para que ésta se haga realidad es mirar el mundo con ojos muy abiertos de modo que se puedan ver las desigualdades existentes y poder dar una respuesta desde la esperanza.

Tal y como consta en el Plan Andaluz de Educación para la Cultura de Paz y Noviolencia (2001:7), la Cultura de Paz fue definida en 1989 en el Congreso Internacional de Yamoussoukro y adoptada en el Programa de la UNESCO en 1995. Pronto se convertiría en un movimiento mundial con la implicación directa de amplios sectores de la sociedad en todos los continentes lo que motivó que Naciones Unidas proclamara el año 2000 como Año Internacional de la Cultura de la Paz, y decidiera la proclamación del Decenio Internacional de la promoción de una Cultura de Paz y No-violencia (2001-2010).

A nuestro juicio, una alternativa similar al Plan Andaluz de Educación para la Cultura de Paz y No-violencia se puede aplicar en EEUU ya que los principios que definen y determinan la Cultura de la Paz tal y como consta en las páginas 7 y 8 de dicho plan se pueden aplicar en cualquier parte del mundo: respetar la vida, rechazar la violencia, compartir con los demás, escuchar para entender, conservar el planeta y redescubrir la solidaridad. En la página 8 de dicho plan se pone de manifiesto que la cultura de la paz ha de conseguir metas en ocho esferas:

- Cultura de paz a través de la educación

- Desarrollo económico y social sostenible

- Respeto de todos los derechos humanos

- Igualdad entre hombres y mujeres

- Participación democrática

- Comprensión, tolerancia y solidaridad

- Comunicación participativa y libre circulación de información y conocimientos

- Paz y seguridad internacionales

Aunque este plan no se experimentó en profundidad en el programa de educación bilingüe que es objeto de análisis, algunas ideas que el plan propone están en la base del programa bilingüe como el respeto a las diferentes culturas, la escucha y el rechazo a la violencia. 
María Martínez Lirola ¿ ¿Qué relación guarda la motivación con los problemas de disciplina ...

\section{Conclusiones}

La baja motivación, un marco de valores basado en el materialismo y la superficialidad, la carencia de valores humanos auténticos y la desesperanza general que envolvía a los jóvenes a los que les dimos clase generaba en los adolescentes actitudes agresivas y comportamientos violentos en la familia, en las escuelas e institutos y en la calle.

El hecho de que nuestros alumnos fueran adolescentes con edades comprendidas entre los 15 y los 19 años llevaba consigo que su motivación, uno de los factores principales que influyen en el aprendizaje de una lengua, apenas existiera. Es obvio que la sola presencia física en EEUU no era suficiente para que los alumnos aprendieran lengua inglesa y se interesaran por tener acceso a una nueva cultura.

Para motivar a los alumnos, el profesor ha de establecer relaciones entre la materia y el mundo real, convertir la clase en un entorno confortable así como que los alumnos se consideren agentes totalmente activos en el proceso de enseñanza aprendizaje

El factor edad es determinante en la enseñanza y aprendizaje de L2 para los adolescentes ya que a esta edad, los alumnos no suelen ser receptivos a profundizar en otras lenguas y otras culturas. Una de nuestras conclusiones principales es que, a pesar de que nuestros alumnos estudiaban la lengua inglesa en un contexto natural, la competencia que alcanzaban en esta lengua no se parecía en absoluto al nivel de un nativo, como quizás podría esperarse, debido al rechazo que los alumnos tenían hacia la lengua inglesa y la cultura norteamericana.

Sin duda, es fundamental que los docentes dediquemos nuestra energía no tanto a aplicar medidas disciplinarias sino a prevenir los problemas de disciplina en el aula. El profesor debe hacer un gran esfuerzo por observar a los alumnos constantemente, de modo que si surgen algunos problemas de disciplina, puedan solucionarse rápidamente.

\section{RefEREnCias Bibliográficas}

Baetens Beardsmore, H. (1982). Bilingualism: Basic Principles. Avon: Tieto Ltd.

Charles, C. M. (1991). Building Classroom Discipline: From Models to Practice. Nueva York: Longman.

Consejería de Educación y Ciencia.(2001). Plan Andaluz de Educación para la Cultura de Paz y Noviolencia. Sevilla: Junta de Andalucía.

Di Pietro, R. J. (1978). "Bilingual Education: A New Challenge to Old Priorities", en Paradis (ed.), Aspects of Bilingualism. Columbia, S.C: Hornbeam Press, pp. 101-109.

Ellis, R. (1994). The Study of Second Language Acquisition. Oxford: O.U.P.

Fishman, J.A. (1976). Bilingual Education, an International Sociological Perspective. Rowley: Newbury House Publishers.

Gardner, R. y Lambert, W. (1959). "Motivational Variables in Second Language Acquisition". Canadian Journal of Psychology 13/4: 266-273.

Gardner, R. y Lambert, W. (1972). Attitudes and Motivation in Second Language Learning. Rowley: Newbury House.

Gass, S.M. y L. Selinker (2001). Second Language Acquisition. An Introductory Course. Mahwaw : Lawrence Erlbaum Associates.

Hamers, J.F. y M. Blanc. (1983). Bilingualité et Bilinguisme. Bruxelles: Pierre Mardaga. 
Krashen, S.D. (1996). Under Attack: The Case Against Bilingual Education. Culver City: Language Education Associates.

Krashen, S.D. y T.D. Terrell (1988). The Natural Approach. Language Acquisition in the Classroom. Hertfordshire: Prentice Hall International.

Lewis, E. G. (1981). Bilingualism and Bilingual Education. Oxford/New York/Toronto/Sydney/ Paris/Frankfurt: Pergamon Press.

Larsen D. N. y W.A. Smalley. (1972). Becoming Bilingual: A Guide to Language Learning. New Canaan, CN: Practical Antrophology.

Martínez Sánchez, A. (2001). "La violencia en la escuela. Propuestas de educación para la paz y la cooperación", en Innovación Educativa 11, 61-83.

Pavón Vázquez V. (2001).“Un estudio sobre la influencia del componente afectivo actitud en la competencia de aprendices adultos de inglés”. En Muñoz, C. (coord.), Trabajos en Lingüística Aplicada. Barcelona: AESLA, 171-178.

Prodromou, L. (1995). “Ain't Misbehavin': Dealing with Discipline”, en Actas de XI Jornadas Pedagógicas para la Enseñanza del Inglés. Granada: Greta, 57-73.

Rodríguez, R. (1998). "Bilingual Education: Outdated and Unrealistic", in Clark, V.P, P.A. Eschholz \& A.F. Rosa (eds.), Language. Readings in Language and Culture. Boston/New York: Bedford/St. Martin's, 483-488. 